LA GRANA:

REVISTA DE

CIENCIAS DE LA VIDA

pISSN:1390-3799; eISSN:1390-8596

http:/ / doi.org/10.17163/lgr.n30.2019.07
Artículo científico / Scientific paper

PRODUCTOS NATURALES

\title{
CARACTERIZACIÓN DEL ACEITE DE LA SEMILLA DE SACHA INCHI (plukenetia volubilis) DEL CANTÓN SAN VICENTE, MANABÍ, ECUADOR, OBTENIDA MEDIANTE PROCESOS NO TÉRMICOS DE EXTRUSIÓN
}

\author{
CHARACTERIZATION OF SACHA INCHI SEED OIL (plukenetia volubilis) FROM \\ "CANTON SAN VICENTE, MANABí, ECUADOR", OBTAINED BY NON-THERMAL \\ EXTRUSION PROCESSES
}

Luis Eduardo Romero Hidalgo $\oplus$, Carlos Jefferson Valdiviezo Rogel $\oplus$ y Stefanie Michelle Bonilla Bermeo(i)

Facultad de Ingeniería Química, Universidad de Guayaquil. Av. Delta y Av. Kennedy Guayaquil, Ecuador.

*Autor para correspondencia: luis.romeroh@ug.edu.ec

Manuscrito recibido el 23 de enero de 2019. Aceptado, tras revisión, el 16 de julio de 2019. Publicado el 1 de septiembre de 2019.

\begin{abstract}
Resumen
Sacha Inchi (Plukenetia Volubilis) es una planta originaria de Perú. El fruto es una semilla oleaginosa la cual posee un alto contiene ácidos grasos polinsaturados, en la cual se destaca el omega $(\alpha) 3$ y 6 . Para la extracción de este aceite se utilizó el método de compresión por extrusión, utilizando un tornillo sinfín lo que hace aumentar la presión de la masa, separando así el aceite contenido dentro de la semilla. Para esto, se utilizó un extractor experimental el cual fue adaptado y puesto en marcha para la obtención de este aceite, extrayéndolo a temperatura ambiente. Con la finalidad de establecer las características fisicoquímicas de la semilla se realizaron análisis de humedad, fibra, ceniza, grasa y proteína. Una vez extraído el aceite se calculó los rendimientos y se realizaron análisis de: índice de acidez, densidad relativa, índice de Yodo, índice de Peróxido y perfil de ácidos grasos. Estos resultados se compararon con análisis realizados al aceite de la misma especie, pero de diferentes zonas de cultivo del Perú, aceite de pescado y oliva, los cuales se los conoce por su alto contenido de ácidos grasos, dejando como resultado que el aceite de Sacha Inchi presenta un alto contenido de ácidos grasos polisaturados y que el método de extracción influye en la calidad del producto.

Palabras clave: Sacha Inchi, extrusión, índice de peróxido, ácido graso insaturado, índice de yodo.
\end{abstract}




\begin{abstract}
Sacha Inchi (Plukenetia Volubilis) (SI) is a plant native from Peru. The fruit of this plant is an oilseed than contains a high content of oil which is rich in unsaturated fatty acids (91.6\%), being one of the seeds that contain this type of fat in higher percentage. For the extraction of this oil, the extrusion method was used, using an endless screw that allows the pressure increase in the dough, separating the oil contained in the seed. For this, an experimental extractor was adapted and put into operation to obtain this oil, extracting it at room temperature. To analyze the physicochemical characteristics of the seed, moisture, fiber, ash, fat and protein analyzes were conducted. Once the oil was obtained, the yields were calculated, and the following analyzes were performed: acid index, relative density, iodine value, peroxide index and fatty acid profile. Those results were compared with the analysis made to olive and fish oil, known for their high content of fatty acids, resulting that Sacha Inchi oil is better in both quality indexes, as in percentage of unsaturated fatty acids.
\end{abstract}

Keywords: Sacha Inchi, extrusion, peroxide index, unsaturated fatty acid, iodine index.

Forma sugerida de citar: $\quad$ Romero H., L.E., Valdiviezo R., C. y Bonilla R., S. (2019). Caracterización del aceite de la semilla de Sacha Inchi (plukenetia volubilis) del cantón San Vicente, Manabí, Ecuador, obtenida mediante procesos no térmicos de extrusión. La Granja: Revista de Ciencias de la Vida. Vol. 30(2):77-87. http:/ / doi.org/10.17163/lgr.n30.2019.07.

IDs Orcid:

Luis Eduardo Romero Hidalgo: https:/ / orcid.org/0000-0002-2417-4836

Carlos Jefferson Valdiviezo Rogel: https:/ / orcid.org/0000-0002-6550-9751

Stefanie Michelle Bonilla Bermeo: https:/ / orcid.org/0000-0002-9391-3698 


\section{Introducción}

Sacha inchi (SI) (Plukenetia volubilis Linnaeus), conocido también como, maní silvestre, maní del Inca, maní sacha o montaña maní, es una planta oleaginosa que pertenece a la familia de las Euphorbiaceae. En un principio y hasta la actualidad ha sido cultivado en las tierras bajas de la amazonía peruana, y siendo plantado durante siglos por la población indígena, ha sido un componente de la dieta de varios grupos nativos de la región (Gutiérrez, Rosada y Jiménez, 2011; Chirinos y col., 2013). Según Muangrat, Veeraphong y Chantee, (2018) el porcentaje de obtención de este aceite prensado a $60{ }^{\circ} \mathrm{C}$ es $37,97 \%$, el cual tiene un porcentaje aproximado de $92 \%$ de ácidos grasos poliinsaturados (AGP) como ácido alfa linolénico (18:3n-3, ácido $\alpha$-linolénico) y ácido linoleico (18:2n-6, ácido linoleico) (Fanali y col., 2011; Cisneros y col., 2014). Este tipo de ácidos grasos presenta una o varias ligaduras entre sus carbonos, y dependiendo de su ubicación se los denomina $\alpha-3,6$ o 9 BADUI (Araujo-Dairiki, Chaves y Dairiki, 2018).

Estos ácidos grasos tienen efectos beneficiosos que incluyen la capacidad de disminuir los niveles de glicéridos, prevenir los trastornos cardiovasculares y una acción antitrombótica; además ciertos experimentos han demostrado que este aceite tiene una alta capacidad antioxidante, ayudando a reducir el daño del ADN debido a la oxidación (Takeyama y Fukushima, 2013). Últimamente el interés por estos nutrientes ha aumentado, demostrándose así que la ingesta de grasa depende de la calidad de esta, es decir del tipo de ácido graso predominante (Carrillo Fernández y col., 2011). De esta forma, el estudio del SI se convierte en un reto tanto como material oleaginoso y también por las funcionalidades biológicas que podrían derivar de su aceite $\mathrm{y} / \mathrm{o}$ de sus extractos (T. y col., 2012).

Por otra parte, el consumo de aceite de oliva (AO) ha incrementado debido a los beneficios de los aceites vegetales por la composición del $\mathrm{AO}$, el cual tiene en su interior una gran cantidad de ácidos grasos monoinsaturados, en particular ácido oleico. Además, de estar presentes el ácido $\alpha$-linolénico $(\alpha-$ 3) y ácido linoleico $(\alpha-6)$ los cuales son requeridos por el cuerpo humano y no puede sintetizar (Piscopo y col., 2016). Actualmente, el aceite de pescado en capsulas es comercializado globalmente debido a que es rico en ácidos grasos poliinsaturados a-3 [40.91\% según Paucar-Menacho y col., (2015)], ácido eicosapentaenoico (EPA) (20: 5, n-3) y ácido docosahexaenoico (DHA) (22: 6, n-3) (Tempel y col., 1990). Sin embargo, la contaminación ambiental ha provocado la acumulación de metales pesados y dioxinas en los peces, y debido a esto se cuestionan los beneficios de obtener ácidos grasos insaturados a partir de peces (Maurer y col., 2012).

Las personas que no pueden comer pescado diariamente o lo hacen con poca frecuencia pueden complementar su ingesta de ácidos grasos $\alpha-3$ con aceites vegetales (Strobel, Jahreis y Kuhnt, 2012). Sin embargo, como estos ácidos grasos se oxidan fácilmente a altas temperaturas, sus potenciales aplicaciones son limitadas. Como tal, el análisis de nuevas fuentes de ácidos grasos $\alpha-3$, sería extremadamente beneficioso desde el punto de vista de la salud humana (Takeyama y Fukushima, 2013).

El objetivo de este estudio es extraer el aceite de SI por un prensado en frío y realizar su caracterización para luego poder comparar los aceites ricos en ácidos grasos insaturados, como son el aceite de pescado y el aceite de oliva.

\section{Materiales y métodos}

\subsection{Proceso de obtención del aceite}

Las semillas de SI (Plukenetia volubilis Linnaeus) procedentes del cantón San Vicente, Manabí, Ecuador, una vez cultivadas se almacenaron herméticamente, luego se procedió a extraer la cascara de la semilla de forma manual y dejarla lista para su análisis y procesos de extracción. Finalmente, para separar la masa seca no lipídica de la parte oleosa se usó un extrusor de marca "Piteba" de origen holandés, en el cual su limitación radica en el uso semillas con contenido de grasas totales de más del $25 \%$ de peso bruto. Según Fanali y col., (2011) la presencia de aceite en la semilla de SI es entre el $37 \%$ al $47 \%$. Esta extracción se realizó a temperatura ambiente, con la finalidad de generar el menor impacto posible a las estructuras insaturadas de los ácidos grasos. Una vez extraído el aceite crudo, el cual presenta sólidos en suspensión debido a que durante el proceso de extrusión ciertas partículas de material sólido son retenidas, se deja reposar unas horas en un recipiente debidamente sellado y sin luz para evitar la oxidación acelerada del mismo; una vez separadas las dos fases (sólida de la seca) se procede a la fil- 
tración de la primera capa con papel filtro estándar, dejando así el aceite listo para su almacenamiento como se aprecia en la Figura 1.

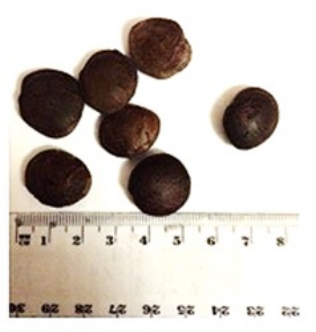

a
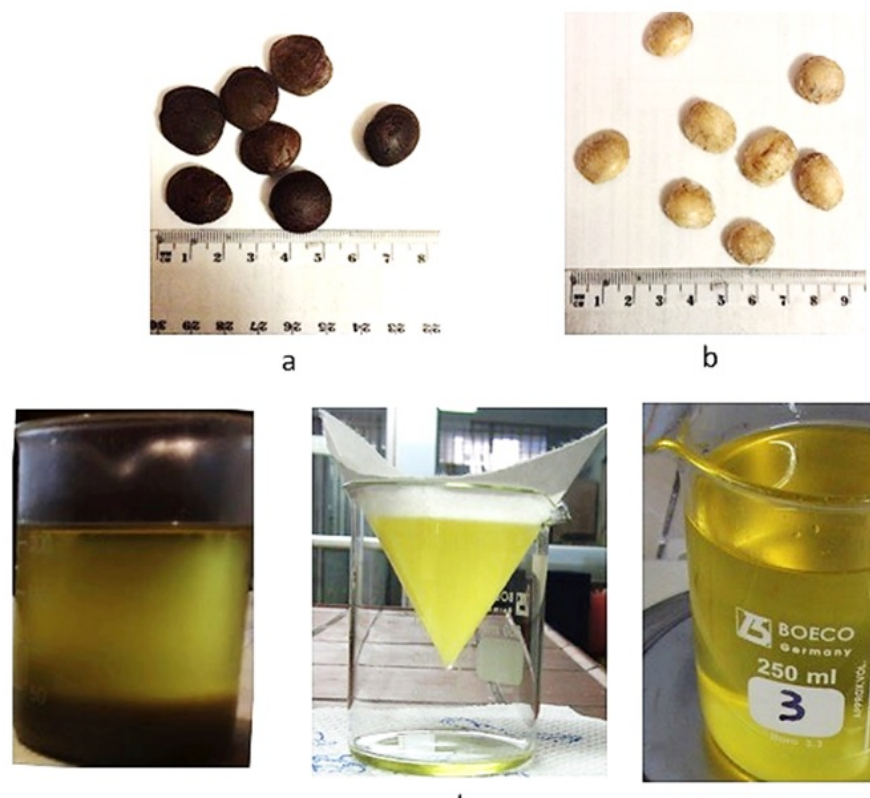

$\mathrm{b}$

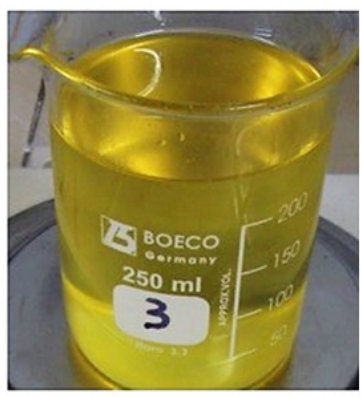

Figura 1. a) Semilla de SI con cascara.

b) Semilla de SI sin cascara.

c) Producto obtenido después de la extrusión.

d)

Filtrado del aceite.

d) Aceite filtrado.

\subsubsection{Análisis físico químico de la semilla de Sacha Inchi}

Cada ensayo se realizó siguiendo los parámetros de la Official Methods of Analysis of AOAC International y de la American Oil Chemists' Society (AOCS). En el caso de necesitar pesar una muestra se usó una balanza AE Adam de máxima capacidad 1000 gramos, la cual estaba debidamente calibrada.

\subsubsection{Porcentaje de humedad}

Siguiendo los lineamientos del método AOCS-94 Cde 13a-63 se pesaron aproximadamente 5 gramos, luego se llevaron a una estufa Thermo Fisher Scientific por aproximadamente 5 horas a $110{ }^{\circ} \mathrm{C}$ para luego volver a ser pesadas. Mediante la Ecuación (1) determinó el porcentaje de humedad existente en la muestra.

$\%$ Humedad $=\left(1-\frac{\text { Peso de muestra seca }(g)}{\text { Peso de muestra húmeda }(g)}\right) * 100$

\subsubsection{Determinación de grasa}

Para este tipo de análisis se siguió el método AOAC 18th 922.06 en el cual se pesaron 2 gramos de la muestra posterior a la extracción la humedad, y mediante la extracción soxhlet se separó la grasa cruda de la muestra y posteriormente se pesó la misma, y utilizando la Ecuación (2) se determinó el porcentaje de grasa total de la muestra.

$\%$ Grasa $=\frac{\text { Peso de grasa obtenida }(g)}{\text { Peso de la muestra de semilla }(g)} * 100$

\subsubsection{Porcentaje de fibra cruda y ceniza}

Para la determinación de fibra se siguieron los parámetros del método AOAC, 2005, 962.09 usando ácido sulfúrico diluido al 1,25\%, solución de hidróxido de sodio al 1,25\%, alcohol etílico al $95 \%$ y éter de petróleo. El porcentaje de ceniza se determinó por el método AOCS-94 Ba 5-4 en el cual se usó una mufla (1) Thermo scientific Lindbergh blue $\mathrm{m}$. 


\subsubsection{Porcentaje de proteína}

Para este tipo de ensayo se utilizó un analizador Flash 2000 de marca Thermo Fisher Scientific, en el cual se pesó 1 miligramo de la muestra y se llevó a la entrada del instrumento, luego se combustionó alrededor de $900^{\circ} \mathrm{C}$ y posteriormente se realizó una columna en forma de serpentín y finalmente se detectaron por un Detector de Conductividad Térmica (TCD), el cual automáticamente brinda los datos de porcentaje de proteína.

\subsection{Análisis físico químico del aceite}

Para estos análisis también se utilizaron los lineamientos descritos en el punto 2.1. En el caso de necesitar pesar una muestra se usó una balanza $\mathrm{AE}$ Adam de máxima capacidad 1000 gramos, la cual estaba debidamente calibrada.

\section{3 Índice de acidez libre}

De conformidad con el método AOCS-94 Cd 3a-63, este se realizó por titulación directa; se pesaron 10 gramos de la muestra de aceite en un Erlenmeyer y se añadieron $50 \mathrm{ml}$ de alcohol etílico, luego se agregaron alrededor de 3 gotas de indicador fenolftaleína. Posteriormente se tituló con una solución de hidróxido de sodio $(\mathrm{NaOH})$ 0,1 N hasta observar el cambio de color a rosa. Finalmente se realizó el cálculo con la Ecuación (3). Donde IA es el índice de acidez, $\mathrm{V}$ es el volumen en $\mathrm{ml}$ de la solución de $\mathrm{NaOH}$ valorada utilizados para neutralizar los ácidos grasos libres de la muestra, $\mathrm{N}$ es la concentración normal de $\mathrm{NaOH}$ y $\mathrm{W}$ el peso en gramos de la muestra de aceite.

$$
I A=\frac{V * N * 56,1}{W}
$$

\subsubsection{Determinación de densidad relativa}

Para este análisis se usó el método AOAC-90 920212. En una probeta debidamente tarada se colocan $100 \mathrm{ml}$ de la muestra de aceite y luego se pesó esta cantidad para así obtener el resultado con la Ecuación (4) obteniendo el mismo en las unidades de $\mathrm{gr} / \mathrm{ml}$.

$$
\% \text { Densidad relativa }=\frac{\text { Peso del aceite }(g)}{100 \mathrm{ml}}
$$

\subsection{2 Índice de peróxido}

Siguiendo los lineamientos del método AOAC-90 965-33, primero se pesaron dos gramos de la muestra de aceite, luego se añadieron $24 \mathrm{ml}$ de solución 1:3 cloroformo-ácido acético, posteriormente se añadieron $0,4 \mathrm{ml}$ de solución yoduro de potasio y $24 \mathrm{ml}$ de agua destilada, se valoró la solución de tiosulfato de sodio con permanganato de potasio al 0,01 $\mathrm{N} \mathrm{y}$ se continuó con la titulación hasta que desapareció el color azul. Se calculó el índice de peróxido con la Ecuación (5) y el resultado arroja miliequivalentes de oxigeno activo por kilogramos de grasa. Donde IP es el índice de peróxido, V son los mililitros de solución valorada de tiosulfato de sodio empleados en el ensayo, $\mathrm{V}$ son los mililitros de solución valorada de tiosulfato de sodio consumidos en el blanco, $\mathrm{N}$ es la normalidad de la disolución de tiosulfato de sodio y $\mathrm{P}$ es el peso en gramos de la muestra. Simultáneamente se realizó un blanco realizando el mismo procedimiento con agua.

$$
I P=\frac{\left(V-V^{\prime}\right) * N * 1000}{P}
$$

Este ensayo se realizó durante 13 días con el propósito de estudiar el avance de la oxidación de este aceite a la intemperie, temperatura ambiente y a la exposición a la luz. Los análisis se llevaron a cabo en los días 1-4-6-8-11-13. Se utilizaron barras de error como método de análisis estadístico.

\subsection{3 Índice de Yodo}

La medición de este parámetro se realizó de acuerdo a los parámetros establecidos en el método AOAC-90 920-159. Primero se pesó 0,1 g de aceite de SI en un Erlenmeyer de $250 \mathrm{ml}$, el aceite se disolvió en $10 \mathrm{ml}$ de cloroformo y $10 \mathrm{ml}$ de la solución de Wijs, luego se dejó reposar 30 minutos en la oscuridad agitándolo ocasionalmente, posteriormente se añadieron $5 \mathrm{ml}$ de solución de yoduro de potasio al $15 \%$, se agitó vigorosamente y se añadieron $100 \mathrm{ml}$ de agua recién hervida y enfriada, lavando cualquier residuo de la solución existente en los bordes.

Finalmente se tituló con tiosulfato de sodio 0,1 N y se empleó almidón como indicador. Paralelamente se realizó un análisis de una muestra en blanco. El cálculo del índice de yodo se realizó con la Ecuación (6). En el cual $V_{s}$ son los mililitros de tiosulfato de sodio usados en el blanco, $V_{m}$ son los mililitros 
de tiosulfato de sodio usados en la muestra y $N$ es la normalidad del tiosulfato de sodio.

$$
\text { Índice de Yodo }=\frac{\left(V_{s}-V_{m}\right) * N * 12,67}{\text { peso de la muestra }}
$$

\subsection{Perfil de acidos grasos}

Se realizó de acuerdo con el procedimiento AOCS Ce 1B-89 usando un cromatógrafo de gases de marca TRACE 1310 Mainframe con una columna capilar Trace ${ }^{\text {TM }}$ TR-FAME 260M137P (25m x 0,32mm x $0.25 \mathrm{um})$. Las condiciones de trabajo se realizaron según describe en Wang y Kakuda, (2018).

\subsection{Análisis estadístico}

Para los análisis fisicoquímicos de la semilla y el aceite se hicieron tres repeticiones de cada uno, y se obtuvieron las medias con sus respectivas desviaciones estándar. Además, se hizo un análisis estadístico usando la prueba ANOVA de Tukey usando el programa de Real Statistics para determinar las posibles diferencias significativas a nivel $p<0,05$. Para la elaboración de gráficos se utilizó el software 'R'.

\section{Resultados y Discusión}

\subsection{Rendimientos en la extracción del acei- te de la semilla de Sacha Inchi}

Como se aprecia en la Tabla 1, se puede observar que tomando 1000 gramos de semilla de SI se pierde gran cantidad de este peso total en la cascara, por lo cual se tomará el peso de la semilla sin cascara como base de cálculo para los demás parámetros. La masa seca (residuo de la extracción) obtenida después de la extracción $(68,08 \%)$ arroja un valor muy alto, por lo cual representa un porcentaje muy importante ya que se puede usar posteriormente. El porcentaje de rendimiento de aceite que se observa en la Tabla 1 es de 26,92 \%, el cual comparado con los resultados obtenidos por Muangrat, Veeraphong y Chantee, (2018) quien obtuvo rendimientos

\subsubsection{Densidad relativa}

El resultado de este parámetro en la Tabla 3 arroja un valor de 0,91 , el cual demuestra que este aceite de aceite entre $37,97 \%$ a $40,63 \%$, se evidencia que el rendimiento en este estudio fue menor, debido a que durante la extracción del aceite no se elevó la temperatura en esta investigación.

\subsection{Análisis fisicoquímico de la semilla de Sacha Inchi}

Como se puede observar en la Tabla 2, esta semilla contiene un alto contenido de aceite: $42,0 \%$ el cual según Wang y Kakuda, (2018) se encuentra en el rango estimado $(33,4 \%-54,3 \%)$. La cantidad de proteína: $29,78 \%$, da a entender que esta semilla después de haber extraído el aceite quedará con un remanente de proteína muy alto. El porcentaje de ceniza es de $2,9 \%$ que es un poco menor según lo reportado por Gutiérrez, Rosada y Jiménez, (2011) el cual obtuvo un porcentaje de $4 \%$. La cantidad de humedad es relativamente baja: 6,72\% ya que se trata de un fruto seco, según James, (1995) se encuentra dentro del rango $0-10 \%$ para procesamiento y almacenamiento sin degradación de microorganismos a los triacilgliceridos. La cantidad de fibra obtenida en este estudio fue de $18 \%$ en base seca, el cual es mayor al reportado por Muangrat, Veeraphong y Chantee, (2018), quien obtuvo un porcentaje de $13,86 \%$ en base seca.

\subsection{Análisis físico químico del aceite de la semilla de Sacha Inchi}

\subsection{1 Índice de Acidez}

Según se observa en la Tabla 3, el índice de acidez de este aceite es 0,38 mg KOH/g. Según FAO/OMS, (2015) no debe ser mayor a $4 \mathrm{mg} \mathrm{NaOH} / g$ para aceites vegetales prensados en frío. La baja acidez de este ácido refleja la escasa refinación y la buena calidad del aceite de SI, en comparación con el aceite de oliva que según Paucar-Menacho y col., (2015) tiene un valor de 1,14 mg NaOH/g, y el aceite de pescado que según Nascimento V. y col., (2015) tiene un valor de 11,72 mg NaOH/g, se puede corroborar la buena calidad de este aceite.

es ligero. Según Gutiérrez, Rosada y Jiménez, (2011) esto se debe a la cantidad de ácidos grasos insaturados presentes en el mismo, comparándolo con el 
Tabla 1. Rendimientos en la obtención del aceite de la semilla de SI.

\begin{tabular}{cccccc}
\hline $\begin{array}{c}\text { Aceite obtenido } \\
(\% \mathbf{p} / \mathbf{p})\end{array}$ & $\begin{array}{c}\text { Sedimentos } \\
(\boldsymbol{\%} \mathbf{p} / \mathbf{p})\end{array}$ & $\begin{array}{c}\text { Residuo de } \\
\text { semilla }(\boldsymbol{\%} \mathbf{p} / \mathbf{p})\end{array}$ & $\begin{array}{c}\text { Semilla sin } \\
\text { cáscara }(\mathbf{g})\end{array}$ & $\begin{array}{c}\text { Cáscara } \\
(\mathbf{g})\end{array}$ & $\begin{array}{c}\text { Semilla con } \\
\text { cáscara }(\mathbf{g})\end{array}$ \\
\hline $26,93 \pm 1,67$ & $3,8 \pm 0,18$ & $68,08 \pm 7,46$ & $653,17 \pm 13,83$ & $347,2 \pm 14,4$ & $1000,3 \pm 0,6$ \\
\hline
\end{tabular}

aceite de oliva que según Paucar-Menacho y col., litud entre estos dos aceites debido a la alta insatu(2015) da un valor de 0,9252, existiendo una simi- ración en ambos.

Tabla 2. Características fisicoquímicas de la semilla de SI

\begin{tabular}{cc}
\hline Característica & Semilla de SI \\
\hline Humedad & $6,72 \pm 0,1$ \\
Grasa & $42,03 \pm 0,2$ \\
Ceniza & $2,9 \pm 0,025$ \\
Fibra & $18,0 \pm 0,095$ \\
Proteína & $29,78 \pm 1,6$ \\
\hline
\end{tabular}

\subsection{3 Índice de Yodo}

Según Muangrat, Veeraphong y Chantee, (2018), esta característica del aceite está relacionada con el grado de insaturación del mismo, que como se puede observar en la Tabla 3 arroja un valor de 192,5 I2/100g. Haciendo una comparación según lo re- portado por Paucar-Menacho y col., (2015) para el aceite de oliva $(56,15 \mathrm{~g}$ I2/100 g) y por Nascimento V. y col., (2015) para el aceite de pescado $(93,92 \mathrm{~g}$ $\mathrm{I} 2 / 100 \mathrm{~g})$, demuestra un valor muy alto en el índice de yodo para el aceite de SI, reflejando así que este aceite tiene una mayor insaturación en comparación a los otros dos aceites.

Tabla 3. Características físicoquímicas del aceite de SI

\begin{tabular}{cccc}
\hline \multirow{2}{*}{ Propiedad } & \multicolumn{3}{c}{ Tipo de aceite } \\
\cline { 2 - 4 } & Sacha Inchi & Oliva & Pescado $^{3}$ \\
\hline $\begin{array}{c}\text { Índice de acidez } \\
(\mathrm{mg} \mathrm{KOH} / \mathrm{g})\end{array}$ & $0,38 \pm 0,02$ & $1,14 \pm 0,037^{1}$ & $11,72 \pm 0,1$ \\
\hline Densidad relativa & 0,91 & $0,9252 \pm 0,0014^{1}$ & $\mathrm{~N} / \mathrm{D}$ \\
\hline $\begin{array}{c}\text { Indice de peróxido } \\
\left(\mathrm{m}-\mathrm{eq} \mathrm{O}_{2} / \mathrm{Kg}\right)\end{array}$ & $5,81 \pm 0,5$ & $6,91 \pm 3,44^{2}$ & $7,22 \pm 1,1$ \\
\hline $\begin{array}{c}\text { Indice de Yodo } \\
\left(I_{2} / 100 \mathrm{~g}\right)\end{array}$ & $192,5 \pm 0,7$ & $56,15 \pm 0,14^{1}$ & $93,92 \pm 0,7$ \\
\hline $\begin{array}{c}{ }^{1}(\text { Paucar-Menacho y col., } 2015),{ }^{2} \text { (Piscopo y col., 2016), }{ }^{3} \text { (Nascimento V. y } \\
\text { col., 2015). }\end{array}$ & & \\
\end{tabular}

\subsection{4 Índice de peróxido}

Según indica Cebi y col., (2017) este ensayo mide directamente la concentración de hidroperóxido, que es el resultado del producto de oxidación primario en la oxidación de productos, que según FAO/OMS, (2015) no debe de exceder de 10 mili- equivalentes de peróxido de oxígeno / $\mathrm{kg}$ en el caso de aceites vegetales y de origen animal. El resultado obtenido en este estudio fue de $5,81 \mathrm{~m}$-eq $O_{2} / \mathrm{Kg}$, el cual arroja un valor menor en comparación al aceite de oliva con un valor de 6,91 m-eq $O_{2} / \mathrm{Kg}$ según Piscopo y col., (2016), y con el aceite de pescado con un valor de 7,22 m-eq $O_{2} / \mathrm{Kg}$ según Nascimento V. 
y col., (2015).

Para conocer que tan vulnerable es el aceite de SI a la oxidación se realizaron los procedimientos descritos en el punto 2.2.3 a temperatura ambiente $\left(25^{\circ} \mathrm{C}\right)$, dando como resultado que este aceite es sensible a desarrollar rancidez si está expuesto prolongadamente a la luz y a la intemperie. Según lo reportado por Takeyama y Fukushima, (2013) quienes expusieron el aceite a una radiación UV de $300 \mathrm{~nm}$ por 15 días y obtuvieron un incremento de la oxi- dación del mismo, llegando a valores de $250 \mathrm{~m}$-eq $\mathrm{O}_{2} / \mathrm{Kg}$. Según lo reportado por Maurer y col., (2012) quienes obtuvieron un valor de $100 \mathrm{~m}$-eq $\mathrm{O}_{2} / \mathrm{Kg}$ a $65^{\circ} \mathrm{C}$ durante 15 días, que es muy diferente en comparación al análisis realizado en esta investigación, el cual fue llevado a cabo a $25^{\circ} \mathrm{C}\left(16 \mathrm{~m}\right.$-eq $\mathrm{O}_{2} / \mathrm{Kg}$ durante 15 días). Todos estos resultados indican que este aceite tiene una gran sensibilidad a la oxidación debido a la exposición a la luz ultravioleta y a la temperatura.

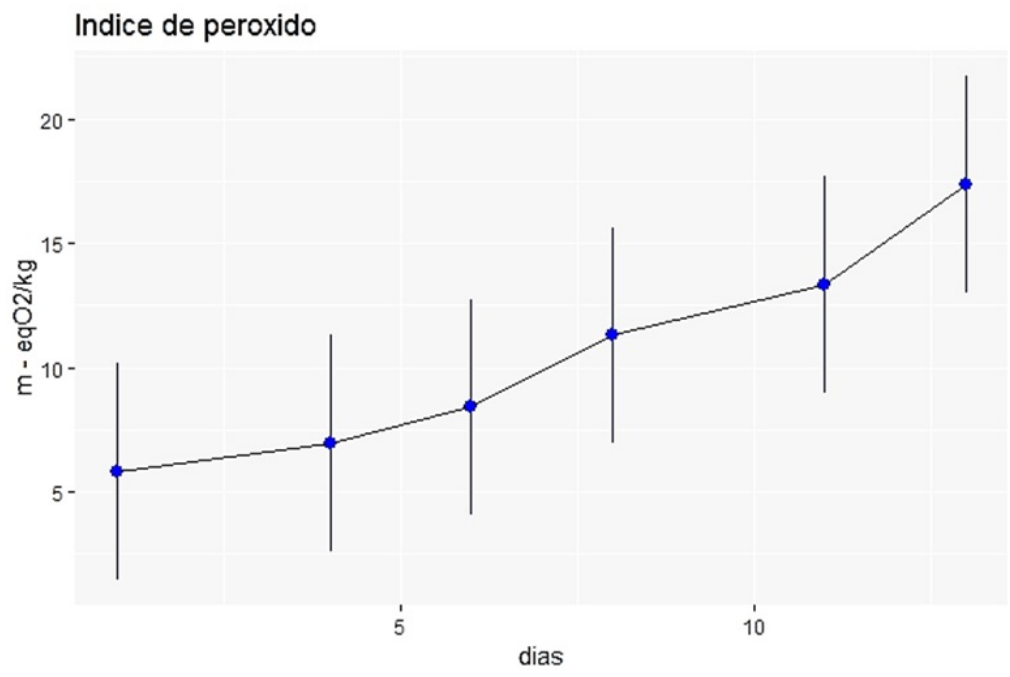

Figura 2. Índice de oxidación (m-eq $\mathrm{O}_{2} / \mathrm{Kg}$ ) vs tiempo (días).

\subsection{Perfil de ácidos grasos}

El perfil de ácidos grasos se puede observar en la Tabla 4. En los tres aceites se puede apreciar el alto contenido de ácidos grasos insaturados, siendo el más alto el de SI $(90,63 \%)$ muy parecido al reportado por Fanali y col., (2011) quienes obtuvieron un valor de $92 \%$. Dentro de ellos el ácido linolénico $\alpha$ $3(38,84 \%)$ es el de mayor presencia, el cual ayuda a disminuir el riesgo de enfermedades cardiovasculares en seres humanos (Araujo-Dairiki, Chaves y Dairiki, 2018). El ácido graso linoleico $\alpha-6$ es el segundo con mayor porcentaje dentro del aceite de SI $(34,67 \%)$, el cual contribuye a la prevención de enfermedades inflamatorias (Saiki y col., 2017) y disminuye la grasa corporal en niños (Racine y col., 2010). Además, comparando con los valores obtenidos en este ensayo con los brindados por Cisneros y col., (2014), se observa que existe una pequeña diferencia en la composición de ácidos grasos, llegando a valores más altos en el ácido linolénico $\alpha-3$ $(48,2 \%)$ y decreciendo en el ácido oleico $\alpha-9(8,9 \%)$. En el ácido linoleico $\alpha-6$ no existe diferencia significativa.

Según Romero Aroca, (2011) el aceite de oliva tiene un porcentaje total de saturados del $15,88 \%$ y para insaturados tiene un porcentaje total del $83,92 \%$, recalcando que el ácido oleico $\alpha-9$ es el de mayor presencia en este tipo de aceites, obteniendo un porcentaje de $71,22 \%$, el cual es estable a la oxidación debido a que es un ácido graso con solo un doble enlace (Paucar-Menacho y col., 2015). Según Paucar-Menacho y col., (2015) el perfil de ácidos grasos del aceite de pescado tiene un porcentaje total de saturados de $37,42 \%$ y para insaturados tiene un porcentaje total del $62,58 \%$. Cabe recalcar que el acido linolénico $\alpha-3$ es el de mayor presencia en 
este tipo de aceite, alcanzando un $40,91 \%$.

Realizando una comparación entre estos tres aceites se puede observar que el aceite de SI brinda una mayor cantidad de ácidos grasos insaturados, los cuales difieren en la cantidad entre acido linolénico $\alpha-3$, linoleico $\alpha-6$ y oleico $\alpha-9$, siendo los dos primeros los de mayor presencia. Se puede afirmar que el aceite de SI tiene un efecto beneficioso para la salud, reduciendo la cantidad de triglicéridos en la sangre, pudiendo de ayuda en el control de ciertas enfermedades como la diabetes mellitus, la obesidad y sirviendo también como posibles agentes citotóxicos para ciertas células tumorales (RodríguezCruz y col., 2005).

Tabla 4. Perfil de ácidos grasos de los aceites.

\begin{tabular}{|c|c|c|c|c|}
\hline \multicolumn{5}{|c|}{ Perfil de ácidos grasos } \\
\hline Composición de acido graso & Sacha Inchi( \%) & Sacha Inchi $(\%)^{1}$ & Oliva $(\%)^{2}$ & Pescado $(\%)^{3}$ \\
\hline $\mathrm{C} 14: 0$ & N.D & N.D & N.D & 10,22 \\
\hline C16:0 & 5,44 & 4,7 & 13,56 & 22,8 \\
\hline C16:1 & N.D & N.D & 1,43 & 10,54 \\
\hline C17:0 & N.D & N.D & 242 & 0,85 \\
\hline C18:0 & 3,94 & 3,3 & 1,86 & 4,4 \\
\hline C18:1 & 17,12 & 8,9 & 71,22 & 9,62 \\
\hline C18:2 & 34,67 & 34,1 & 10,13 & 1,51 \\
\hline C18:3 & 38,84 & 48,2 & 618 & 0,99 \\
\hline $\mathrm{C} 20: 0$ & N.D & N.D & 356 & N.D \\
\hline $\mathrm{C} 20: 5 \mathrm{n} 3$ & N.D & N.D & N.D & 20,28 \\
\hline $\mathrm{C} 22: 6 \mathrm{n} 3$ & N.D & N.D & N.D & 19,64 \\
\hline C20:1 & N.D & N.D & 284 & 2,56 \\
\hline C20:2 & N.D & N.D & 102 & N.D \\
\hline C22:1 & N.D & N.D & N.D & 1,62 \\
\hline Total $\alpha-3$ & 38,84 & 48,2 & 618 & 40,91 \\
\hline Total $\alpha-6$ & 34,67 & 34,1 & 10,13 & 1,51 \\
\hline Total $\alpha-9$ & 17,12 & 8,9 & 71,22 & 9,62 \\
\hline Total Saturados & 9,38 & 8 & 15,88 & 37,42 \\
\hline Total Insaturados & 90,63 & 91,2 & 83,92 & 62,58 \\
\hline Total mono insaturados & 17,12 & 8,9 & 73,17 & 20,16 \\
\hline Total Poli insaturados & 73,51 & 82,3 & 10,75 & 42,42 \\
\hline
\end{tabular}

1 (Cisneros y col., 2014), ${ }^{2}$ (Romero Aroca, 2011), ${ }^{3}$ (Paucar-Menacho y col., 2015).

\section{Conclusiones}

El porcentaje de extracción de aceite por extrusión $(26,92 \%)$ comparado al obtenido en los análisis fisicoquímicos $(42,03 \%)$ demuestra que este método de extracción tiene un rendimiento bajo, teniendo como alternativa otros tipos de extracción para obtener más aceite. La semilla de SI tiene un alto contenido de aceite en su interior, y gracias a los estudios realizados en este ensayo se logró determinar que contiene un alto contenido de ácidos grasos insaturados $(90,63 \%)$, comparándolo con los resultados obtenidos con semillas provenientes de Perú, se observa una ligera varianza en los ácidos grasos linolénico $(9,36 \%$ de diferencia) y oleico $(8,22 \%$ de diferencia), llegando a concluir que la composición de ácidos grasos poliinsaturados y monoinsaturados varía dependiendo de la procedencia de la semilla.

Al compararlo con los aceites de oliva y pescado los cuales comúnmente se conocen por su alto nivel de instauración, se observa que este tiene un nivel más alto de ácidos grasos poliinsaturados: $62,76 \%$ de diferencia en comparación con el aceite de oliva y 31,09\% de diferencia en comparación con el aceite 
de pescado. Sin embargo, el aceite de oliva es rico en ácido oleico $\alpha-9$ y el aceite de pescado en ácido linolénico $\alpha-3$, los cuales son beneficiosos dependiendo de la necesidad del consumidor.

Al realizar un análisis fisicoquímico de los tres aceites se pudo determinar que la calidad del aceite de SI es mayor en comparación a los otros dos aceites, al ser un producto menos procesado y más ligero. Debido al alto contenido de proteína presente en el aceite de SI, se recomienda realizar un estudio posterior para obtener un posible producto a partir del residuo después de la extracción de aceite.

\section{Referencias}

Araujo-Dairiki, T., F. Chaves y J. Dairiki (2018). «Seeds of sacha inchi (Plukenetia volubilis, Euphorbiaceae) as a feed ingredient for juvenile tambaqui, Colossoma macropomum, and matrinxã, Brycon amazonicus (Characidae)». En: Acta Amazonica 48.1, 32-37. Online:https: / / bit.ly / 2YTCzL4.

Carrillo Fernández, L. y col. (2011). «Grasas de la dieta y salud cardiovascular». En: Clínica e Investigación en Arteriosclerosis 23, 1-36. Online:https: / / bit.ly/2KnftZl.

Cebi, N. y col. (2017). «Prediction of peroxide value in omega-3 rich microalgae oil by ATRFTIR spectroscopy combined with chemometrics». En: Food chemistry 225, 188-196. Online:https:/ / bit.ly/2YzxyMG.

Chirinos, R. y col. (2013). "Sacha inchi (Plukenetia volubilis): a seed source of polyunsaturated fatty acids, tocopherols, phytosterols, phenolic compounds and antioxidant capacity». En: Food chemistry 141.3, 1732-1739. Online:https: / / bit. ly/2ZLntsC.

Cisneros, F. y col. (2014). «Chemical composition, oxidative stability and antioxidant capacity of oil extracted from roasted seeds of Sacha-Inchi (Plukenetia volubilis L.)» En: Journal of agricultural and food chemistry 62.22, 5191-5197. Online:https://bit.ly/2ZHDkrV.

FAO/OMS (2015). «Norma para aceites vegetales especificados, Codex Alimentarius.» En: Normas internacionales de los alimentos, 1-14. Online:https: / /bit.ly/2M79PN9.

Fanali, C. y col. (2011). «Chemical characterization of Sacha Inchi (Plukenetia volubilis L.) oil». En:
Journal of agricultural and food chemistry 59.24, 13043-13049. Online:https:/ / bit.ly /2K1W1Mw.

Gutiérrez, L., L. Rosada y A. Jiménez (2011). «Chemical composition of Sacha Inchi (Plukenetia volubilis L.) seeds and characteristics of their lipid fraction». En: Grasas y aceites 62.1, 76-83. Online:Online: https://bit.ly/2yKSWPs.

James, C. (1995). Analytical Chemistry of Foods. Vol. Online:https:/ / bit.ly/2GVhR7E. New York: Chapman y Hall publishers.

Maurer, N. y col. (2012). "Characterization and authentication of a novel vegetable source of omega-3 fatty acids, sacha inchi (Plukenetia volubilis L.) oil». En: Food chemistry 134.2, 1173-1180. Online:https:/ / bit.ly/2GT985G.

Muangrat, R., P. Veeraphong y N. Chantee (2018). «Screw press extraction of Sacha inchi seeds: Oil yield and its chemical composition and antioxidant properties». En: Journal of food processing and preservation 42.6, 1-10. Online:https: / / bit.ly / 2GTgiqG.

Nascimento V., L.V. y col. (2015). «Characterization of a hydrolyzed oil obtained from fish waste for nutraceutical application». En: Food Science and Technology 35.2, 321-325. Online:https: / / bit.ly / 2GWTyWK.

Paucar-Menacho, L. M. y col. (2015). «Comparative study of physical-chemical features of sacha inchi oil (Plukenetia volubilis 1.), olive oil (Olea europaea) and fish oil». En: Scientia agropecuaria 6.4, 279-290. Online:https:/ / bit.ly/2MOwYDw.

Piscopo, A. y col. (2016). "Characterization of monovarietal olive oils obtained from mills of Calabria region (Southern Italy)». En: Food chemistry 213, 313-318. Online:Online: https: / / bit.ly / 2YULHPE.

Racine, N.M. y col. (2010). «Effect of conjugated linoleic acid on body fat accretion in overweight or obese children». En: The American journal of clinical nutrition 91.5, 1157-1164. Online:https:/ / bit.ly/2TemiPX.

Rodríguez-Cruz, Maricela y col. (2005). «Mecanismos moleculares de acción de los ácidos grasos poliinsaturados y sus beneficios en la salud». En: Revista de investigación clínica 57.3, 457-472. Online:https:/ / bit.ly/2MOjc40.

Romero Aroca, A.J. (2011). «Caracterización y diferenciación de los aceites vírgenes de oliva de la comarca del Priorat (Tarragona) dentro del mercado global de aceites de la varie- 
dad'Arbequina'». Tesis doct. Online:https://bit. ly/2YwJqyV: Universitat de Lleida.

Saiki, P. y col. (2017). «The anti-inflammatory effect of Agaricus brasiliensis is partly due to its linoleic acid content». En: Food E function 8.11, 4150-4158. Online:https://rsc.li/2yNoXXb.

Strobel, C., G. Jahreis y K. Kuhnt (2012). «Survey of n-3 and n-6 polyunsaturated fatty acids in fish and fish products». En: Lipids in Health and Disease 11.1, 144. Online:https://bit.ly/2YUifgN.

T., D. L. Castaño y col. (2012). «Fatty acid composition of Inca peanut (Plukenetia volúbilis linneo) and its relationship with vegetal bioactivity». En: Revista Chilena de Nutrición 39.1, 45-52. Online:https://bit.ly/31rIJE8.
Takeyama, E. y M. Fukushima (2013). «Physicochemical properties of Plukenetia volubilis L. seeds and oxidative stability of cold-pressed oil (green nut oil)». En: Food Science and Technology Research 19.5, 875-882. Online:https:/ / bit.ly/2KCNfJf.

Tempel, H. van der y col. (1990). «Effects of fish oil supplementation in rheumatoid arthritis.» En: Annals of the rheumatic diseases 49.2, 76-80. Online:https://bit.ly/2TgXW7X.

Wang S.; Zhu, F. y Yukio Kakuda (2018). «Sacha inchi (Plukenetia volubilis L.): Nutritional composition, biological activity, and uses». En: Food chemistry 265, 316-328. Online:https: / / bit.ly / 2GTbrpm. 